\title{
Schriftarten, Abkürzungen und Siglen im Kommentar
}

\begin{tabular}{|c|c|}
\hline kursiv & Editortext \\
\hline Sperrung & Hervorhebung im Editortext \\
\hline$A$ & Eigenhändige oder autorisierte Abschrift von $\mathrm{H}$ \\
\hline$A b b$. & Abbildung \\
\hline Anm. & Anmerkung \\
\hline$B d, B d e$ & Band, Bände \\
\hline bes. & besonders \\
\hline$B l$. & Blatt \\
\hline $\mathrm{cm}$ & Zentimeter \\
\hline$D$ & textgeschichtlich bedeutsamer Druck \\
\hline Diss. & Dissertation \\
\hline$d t$. & deutsch \\
\hline E & Erstdruck \\
\hline$e b d$. & ebenda \\
\hline egh. & Goethe eigenhändig \\
\hline eigentl. & eigentlich \\
\hline engl. & englisch \\
\hline$f ., f f$. & folgende \\
\hline franz. & französisch \\
\hline geb. & geboren \\
\hline gen. & genannt \\
\hline gest. & gestorben \\
\hline griech. & griechisch \\
\hline$H$ & $\begin{array}{l}\text { Handschrift; in der Überlieferung der Briefe Goethes: } \\
\text { behändigte Ausfertigung, eigenhändig oder diktiert }\end{array}$ \\
\hline$h$ & Abschrift von $H$ (nicht autorisiert) \\
\hline$H$. & Heft \\
\hline$H d$ & Hand \\
\hline $\operatorname{Inv} \cdot-\mathrm{N} r$ & Inventar-Nummer \\
\hline ital. & italienisch \\
\hline$J g$ & Jahrgang \\
\hline$K$ & Konzepthandschrift \\
\hline$k$ & Abschrift von $K$ (nicht autorisiert) \\
\hline km & Kilometer \\
\hline
\end{tabular}


lat.

$m$

mhd.

$\mathrm{mm}$

N. F.

$\mathrm{Nr}$

o. J.

o. $\mathrm{Nr}$

o. $\mathrm{O}$.

o. $S$.

$r$

Rs.

$s$.

$S$.

Sign.

$\mathrm{Slg}$

Sp.

Tgb.

T., Tle

u. a.

$v$

V.

v. Chr.

Var.

vgl.

Vs.

I

$\ell$

FDH/FGM

$G M D$

GSA lateinisch

Meter

mittelhochdeutsch

Millimeter

Neue Folge

Nummer

ohne Jahresangabe

ohne Nummerierung

ohne Ortsangabe

ohne Seitenzählung

recto (Blattvorderseite)

Rückseite

siehe

Seite

Signatur

Sammlung

Spalte

Tagebuch

Teil, Teile

unter anderem, unter anderen

verso (Blattrückseite)

Vers, Verse

vor Christus

Variante im edierten Text

vergleiche

Vorderseite

Absatzzeichen in den Lesarten und in Zitaten

Abbrechungszeichen in Zitaten

Freies Deutsches Hochstift/Frankfurter GoetheMuseum

Goethe-Museum Düsseldorf, Anton-und-Katharina-Kippenberg-Stiftung

Klassik Stiftung Weimar/Goethe- und SchillerArchiv 
$H A A B$

Klassik Stiftung Weimar/Herzogin Anna Amalia Bibliothek

KSW Klassik Stiftung Weimar

LATh-HStA Weimar Landesarchiv Thüringen-Hauptstaatsarchiv Weimar

$S B B / S P K$

Staatsbibliothek zu Berlin - Stiftung Preußischer Kulturbesitz

SNM/DLA Marbach Schiller-Nationalmuseum - Deutsches Literaturarchiv Marbach am Neckar

St $A$ Staatsarchiv

ThULB Thüringer Universitäts- und Landesbibliothek

$U A$ Universitätsarchiv

$U B$ Universitätsbibliothek 